\title{
Design of Antennas for a Rectenna System of Wireless Power Transfer in the LTE/WLAN Frequency Band
}

\author{
Settapong Malisuwan, Noppadol Tiamnara, and Nattakit Suriyakrai
}

\begin{abstract}
The Wireless Power Transfer (WPT) system has enabled numerous applications in clean energy applications since their major goal is to recycle the ambient microwave energy. It is enough to drive low power consumption devices. In such system, microstrip antennas are utilized as the main component for collecting the electromagnetic energy. Antenna design is important in the proposed rectenna. The antenna absorbs the incident microwave power, and the rectifier converts it into a useful electric power. In this paper, we focus on the design of two microstrip antennas with resonant frequency of $1.88 \mathrm{GHz}$ and $2.45 \mathrm{GHz}$ to utilize in the WPT system for LTE/WLAN low power devices. The maximum return loss for each of microstrip antennas was simulated by MATLAB programming. The conversion efficiency of the fabricated antennas was measured as $38 \%$ and $35 \%$. The results of this research can be applied in other mobile technologies.
\end{abstract}

Index Terms-WPT, rectenna, microstrip antenna, LTE/WLAN, low power.

\section{INTRODUCTION}

Since 1960s, wireless power transmission technology via microwave has been undergoing development [1] Scientists have focused on converting deep space solar energy into high power microwave energy. The concept is based upon microwave energy that is transmitted to earth surface via high gain parabolic reflector and received and converted to DC power which comprises to a rectenna [2]. It is strongly believed that within the next decades, this phenomenon will become a solution to the world's energy crisis and will become a source of energy especially for high-populated developing countries that cannot afford conventional energy sources.

The wireless energy transfer is when electrical energy is transmitted from a power source to an electrical load, with the absence of wires. Wireless transmission is advantageous when instant and continuous energy transfer is required. When compared to wired connections, wireless power transmission is better since wired connections are inconvenient, hazardous, or could even be impossible [3]. Though the physics of both are related, it is different on purpose of transferring images such as television broadband, where the percentage of the received power is only important if it becomes too low to successfully recover the signal [4]. With wireless energy transfer, efficiency is the most

Manuscript received March 5, 2015; revised December 20, 2015. This research paper is financially supported by National Broadcasting and Telecommunications Commission, Bangkok, Thailand.

The authors are with National Broadcasting and Telecommunications Commission Bangkok, Thailand (e-mail: settapong.m@nbtc.go.th, noppadol.m@nbtc.go.th, nattakit.s@nbtc.go.th). parameter and creates important differences in this technology [1].

Rectenna is known as rectifying antenna [5]. It is a type of antenna that rectifies the incoming electromagnetic waves into DC current. A rectenna physical structure is such that it is made of a mesh of dipoles and diodes for collecting microwave energy from a transmitter and then converted it into electric energy [4]. The elements are commonly arranged in a mesh pattern giving a different and distinct appearance from most antennas. A simplified form of rectenna can be constructed from schottky diode placed between antenna dipoles. Antennas are the backbone in the wireless communication without which the world would not have reached an age with such advanced technological innovations [4], [6].

Rectennas are also used both radio-frequency identification (RFID) and electric vehicles. For RFID, the two main components are tag (transponder), and a reader (interrogator). The tag transponder has electronically stored data, and the reader reads the stored data of the tag. The tag represents a rectenna system since it mainly consists of an antenna, a diode and a microchip.

A rectenna is a part of wireless power transfer system (WPT), which composes of three main subsystems: a transmitter, a travelling medium (i.e., free space), and a receiver, as shown in Fig. 1. The function of the first part is it acts as a transmitter to convert DC power to microwaves using a transmitting circuit and a transmitting antenna.

The microwaves are transmitted via the antenna and it travels across free space towards the receiver which is in the third block. The receiver then converts the microwaves to AC power using the receiving antenna. Further, the captured AC power is then converted back to DC using the rectifying circuit. Therefore, a rectenna is named after its main components.

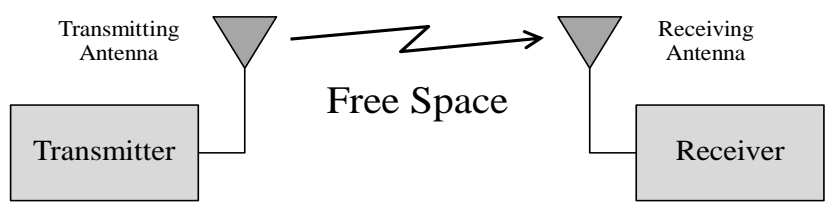

Fig. 1. Wireless power transfer (WPT) system.

As shown in Fig. 2, the AC power has channeled maximally from the antenna through the RF filter, a diode (i.e., schottky diode) converts the collected AC power to DC. To ensure that AC power does not reach the load, a DC filter is inserted after the diode which mainly consists of a shunt capacitor that acts as a short circuit for higher frequencies. Then, a resistive load is used to consume the DC power.

RF energy can be collected from mobile phones that are in close proximity. Therefore, it is capable of providing 
power-on-demand for short-range sensing applications. Other sources of RF energy such as Wi-Fi routers and wireless end devices (i.e., Laptops) are also beyond satisfactory.

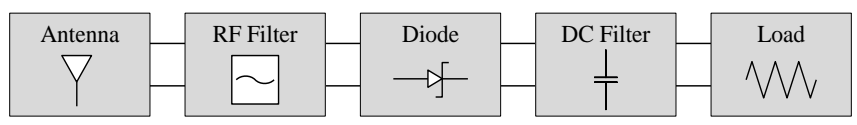

Fig. 2. A rectenna system using an antenna as the primary collector.

An antenna's purpose is for transmission and/or reception of data via different frequency ranges. The frequency ranges and power at different frequency spectrum from $10 \mathrm{kHz}$ to $30 \mathrm{MHz}$ is explained in more detail in [5]. The output power of RF devices is limited due to safety and health concern offered to EM radiation by regulations such as Federal Communications Commission (FCC), USA [6].

Various methods on microwave energy recycling using rectifying antenna system with an antenna have been proposed in many past literatures [7]-[11].

In this paper, we focus on the microstrip antenna design of a rectenna for WPT system for LTE/WLAN with resonant frequency of $1.88 \mathrm{GHz}$ (TDD-LTE band number 35) and $2.45 \mathrm{GHz}$ (ISM band).

\section{RECTENNA SySTEM: MiCROSTRIP ANTENNA DESIGN}

An antenna is one of the fundamental components in any rectenna system since it is the primary energy source that feeds the rectification circuit. Microstrip-type antennas are widely used in communication systems due to their lightweight, low cost, ease of fabrication, low profile, conformability to planar and non-planer surfaces and mechanical robustness. However, microstrip antennas suffer from various drawbacks that limit their applications significantly such as narrow bandwidth, low power handling capability, high feed network losses and high cross [12]. A great deal of research has been aimed at improving antenna characteristics and overcoming the aforementioned drawbacks of microstrip antennas. The advancements in recent antenna technology and the emergence of new concepts such as metamaterials have overcome most of these drawbacks or at least mitigated them to a certain extent. Therefore, antennas in the majority of rectenna systems if not all are of a microstrip type.

A microstrip antenna consist of a dielectric substrate which is in between the radiating patch which is on top and the ground plane which is on the other side as illustrated in Fig. 3 The patch is usually made of gold or copper (conducting material) and the shape varies. The radiating patch and the feed lines are usually photo etched on the dielectric substrate [13].

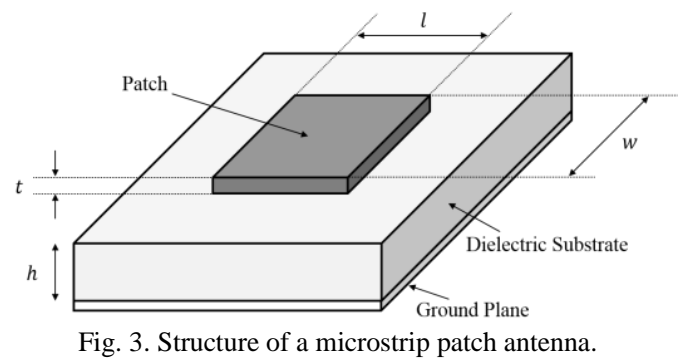

To decrease the complexity of the analysis, the patch is generally square, rectangular, circular, triangular and elliptical or some other common shape. For a rectangular patch, the length $L$ of the patch is usually in the range of $0.3333 \lambda_{o}<L<0.5 \lambda_{o}$, where $\lambda_{o}$ is the free space wavelength. The patch is proposed is very thin such that $t<<\lambda_{o}$ (where $t$ is the patch thickness). The height $h$ of the substrate is usually $0.003 \lambda_{o} \leq h \leq 0.05 \lambda_{o}$. The dielectric constant of the substrate $\epsilon r$ is typically in the range $2.2 \leq$ $\epsilon_{r} \leq 12$ [13], [14].

Choosing the proper feeding configuration is important to achieve better impedance matching to the rectification circuit. Here, the microstrip line feeding mechanism is used since both the antenna and the rectification circuit can be easily fabricated in one plane.

The incident electromagnetic waves with varied polarizations can be exploited more efficiently by antennas with elliptical or circular polarization. Therefore, this research focused on using circularly polarized (CP) microstrip antennas. Rectennas that utilize CP antennas have the advantage of constant output voltage, which is essential in applications where the connected load is rated at certain voltage levels. Furthermore, CP antennas can receive the incoming signal regardless of their position. $\mathrm{CP}$ can be obtained with a single feed by truncating the corners of a patch or by slightly perturbing some segments of the antenna.

Basically, the received power of the system can be increased by expanding the aperture of the antenna. Therefore, in this research, we propose to design two microstrip antennas and each of antennas covers the LTE and WLAN operating frequencies, respectively.

\section{A. Antenna Design for TDD-LTE $1.88 \mathrm{GHz}$}

With the interest in TDD-LTE, there are several unpaired frequency allocations as shown in Table I. The TDD-LTE bands are unpaired because the uplink and downlink share the same frequency, being time multiplexed [15].

TABLE I: TDD-LTE BANDS AND FREQUENCIES [15]

\begin{tabular}{ccc}
\hline \hline LTE band number & Allocation $(\mathrm{MHz})$ & Width of band $(\mathrm{MHz})$ \\
\hline 33 & $1900-1920$ & 20 \\
34 & $2010-2025$ & 15 \\
35 & $1850-1910$ & 60 \\
36 & $1930-1990$ & 60 \\
37 & $1910-1930$ & 20 \\
38 & $2570-2620$ & 50 \\
39 & $1880-1920$ & 40 \\
40 & $2300-2400$ & 100 \\
41 & $2496-2690$ & 194 \\
42 & $3400-3600$ & 200 \\
43 & $3600-3800$ & 200 \\
44 & $703-803$ & 100 \\
\hline \hline
\end{tabular}

$\mathrm{L}$

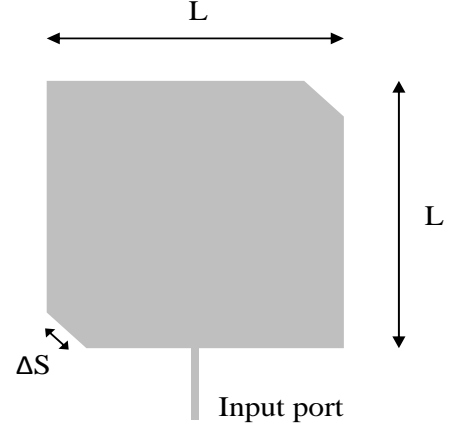

Fig. 4. Circularly/elliptically-polarized square microstrip antenna: for LTE. 
Circular polarization is attainable by cutting off the diagonal corners of a square microstrip as shown in Fig. 4 [16]. In this research the microstrip antenna was designed so that the polarization is nearly circular.

The resonance frequency $f_{r}$ is a function of the effective length of the antenna, which can be determined using the following:

$$
f_{r}=\frac{1}{2 L_{e f f} \sqrt{\mu_{0} \varepsilon_{0}} \sqrt{\varepsilon_{\text {reff }}}}=\frac{V_{0}}{2(L+\Delta L) \sqrt{\varepsilon_{\text {reff }}}}
$$

where $V_{0}$ is the speed of light, $\epsilon_{r}$ is the dielectric constant, and $f_{r}$ is the resonance frequency of the antenna. The extended incremental length $\Delta L$ due to the fringing effect can be found from

$$
\Delta L=0.412(h) \frac{\left(\epsilon_{\text {reff }}+0.3\right)\left(\frac{W}{h}+0.264\right)}{\left(\epsilon_{\text {reff }}-0.258\right)\left(\frac{W}{h}+0.8\right)}
$$

where $h$ is the height of the substrate, and the effective dielectric constant $\epsilon_{\text {reff }}$ is given by the following:

$$
\epsilon_{r e f f}=\frac{\left(\epsilon_{r}+1\right)}{2}+\frac{\left(\epsilon_{r}-1\right)}{2}\left[1+12 \frac{h}{W}\right]_{\text {for } W / h>1}^{-\frac{1}{2}}
$$

Therefore the length of the antenna can be derived as

$$
L=\frac{V_{0}}{2 f_{r} \sqrt{\varepsilon_{r e f f}}}-2 \Delta L
$$

Right triangles at the diagonal corners of the antenna are cut off, where the length $c$ of the two sides of the triangle is equal, and $c$ is calculated using [17].

$$
c=\sqrt{\Delta S}
$$

$\Delta S$ is the area of the triangle, which can be stated as

$$
\frac{\Delta S}{S}=\frac{1}{2 Q}
$$

where $S$ is the area of the antenna patch; $Q$ is the quality factor of the antenna patch.

To determine the quality factor of the antenna $Q$, the effective loss tangent $\delta_{\text {eff }}$ as stated below is needed [8].

$$
\delta_{\text {eff }}=\frac{1}{Q}
$$

$\delta_{\text {eff }}$ can be determined using

$$
\delta_{\text {eff }}=\delta+\frac{\Delta}{h}+\frac{P_{r}}{2 \omega_{0} w_{e}}
$$

where $\Delta$ denotes skin depth and defined by

$$
\Delta=503 \sqrt{\frac{\rho}{\mu_{r} f_{r}}}
$$

where $\rho=$ conductor resistivity; $f_{r}=$ resonance frequency; $\mu_{r}=$ medium permeability.
The electrical energy $w_{e}$ at resonance is

$$
w_{e}=\frac{\varepsilon_{0} \varepsilon_{r} a b V_{0}^{2}}{8 h}
$$

where $V_{0}=$ antenna output voltage

The radiated power $P_{r}$ can be calculated using [16].

$$
P_{r}=\frac{V_{0}^{2} A \pi^{5}}{R_{0} 192}\left[(1-B)\left(1-\frac{A}{15}+\frac{A^{2}}{420}\right)+\frac{B^{2}}{5}\left(2-\frac{A}{7}+\frac{A^{2}}{189}\right)\right]
$$

where $R_{0}=$ intrinsic impedance of free-space; with $A=$ $\left[\pi(a+2 \Delta a) / \lambda_{0}\right]^{2}$ and $B=\left[\pi(b+2 \Delta b) / \lambda_{0}\right]^{2}$ and since a square antenna is used, $a=b=L$.

The dielectric material of the substrate selected for this design is FR-4, which has a dielectric constant $\left(\varepsilon_{r}\right)$ of the 4.4 and a loss tangent equal to 0.017 . The dielectric constant of the substrate material is an important design parameter. A low dielectric constant of the substrate material is used in the prototype design because it gives better efficiency, a higher bandwidth, and a low quality factor $Q$. The low value of the dielectric constant increases the radiated power. The design has a patch size independent of the dielectric constant. Therefore, the reduction in the patch size is accomplished by using a higher dielectric constant and FR-4 is good in this agreement. The small loss tangent was neglected in the simulation. Table II shows the calculated dimensions of the antenna [18].

TABLE II: DIMENSION OF A CP SQUARE MICROSTRIP ANTENNA WITH

\begin{tabular}{cc}
\multicolumn{2}{c}{ 1.88GHz FREQUENCY BAND } \\
\hline \hline Parameter & Value $(\mathrm{mm})$ \\
\hline$\Delta S$ & 3.19 \\
$L$ & 39.75 \\
$h$ & 0.8 \\
\hline \hline
\end{tabular}

Based on the simulation results by using MATLAB programming, the proposed antenna resonates at $1.88 \mathrm{GHz}$ with $-24 \mathrm{~dB}$ return loss. The bandwidth is $180 \mathrm{MHz}$ which covers the TDD-LTE band number 35 as indicated in Fig. 5.

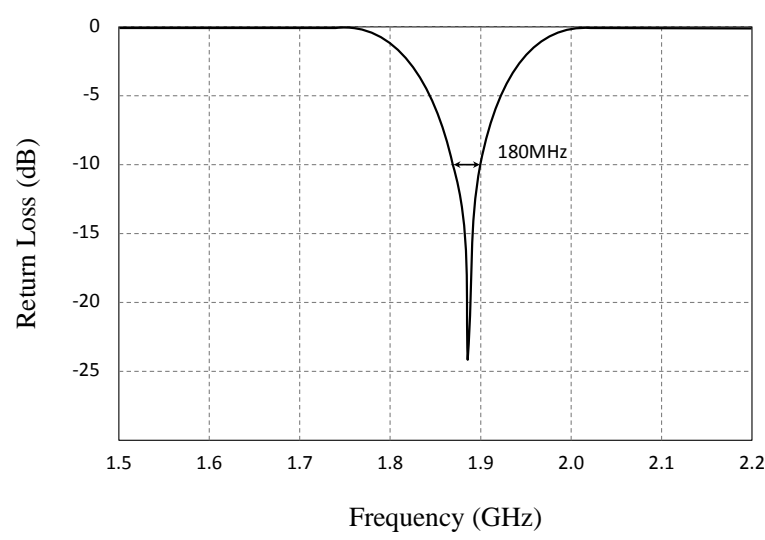

Fig. 5. Return loss of the proposed TDD-LTE $1.88 \mathrm{GHz}$ antenna.

\section{B. Antenna design for WLAN $2.45 \mathrm{GHz}$}

In this part, we design a microstrip antenna to operate at $2.45 \mathrm{GHz}$ frequency range.

A patch antenna is a resonance-based structure having a resonance frequency that depends on the various dimensions of the antenna, as shown in Fig. 6. The width $W$ and the 
length $L$ can be found using the following relation [16].

$$
\begin{gathered}
W=\frac{V_{0}}{2 f_{r}} \sqrt{\frac{2}{\epsilon_{r}+1}} \\
L=\frac{\lambda}{2}-2 \Delta L,
\end{gathered}
$$

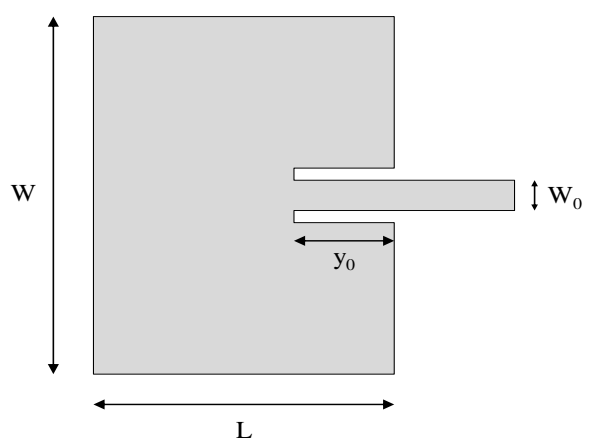

Fig. 6. Circular-polarized square microstrip antenna for WLAN.

In Fig. 6, the feed position $y_{0}$ and the width of the transmission line $W_{0}$ can be found using a model presented in [14].

To find the antenna dimensions, we use MATLAB programming to solve all essential equations at operating frequency of $2.45 \mathrm{GHz}$. Table III summarizes the results of all the antenna dimensions. The proposed antenna in this part was designed using FR4 epoxy dielectric substrate with $\varepsilon_{r}=4.4$.

TABLE III: DIMENSION OF A CP SQUARE MICROSTRIP ANTENNA WITH

\begin{tabular}{cc}
$2.45 \mathrm{GHZ}$ FREQUENCY BAND \\
\hline \hline Parameter & Value $(\mathrm{mm})$ \\
\hline$L$ & 30.2 \\
$W$ & 38.5 \\
$\mathrm{y}_{0}$ & 10.9 \\
$\mathrm{~W}_{0}$ & 2.95 \\
$h$ & 0.8 \\
\hline \hline
\end{tabular}

As shown in Fig. 7, the proposed antenna resonates at $2.45 \mathrm{GHz}$ with $-25 \mathrm{~dB}$ return loss. The bandwidth is $80 \mathrm{MHz}$ which is enough to cover the WLAN ISM frequency band.

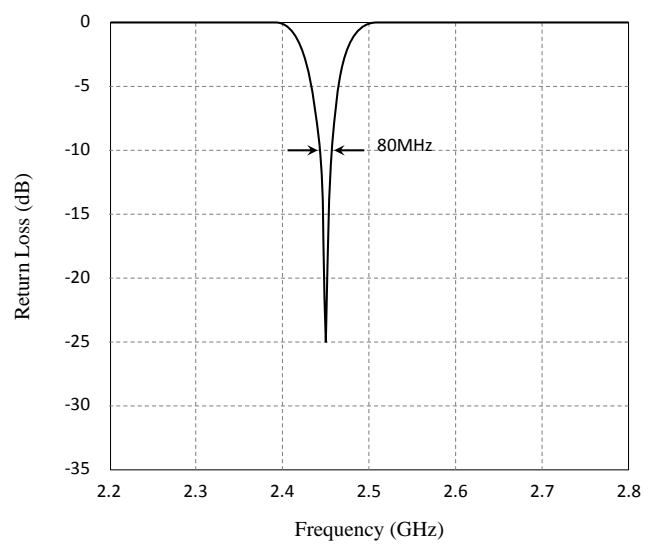

Fig. 7. Return loss of the CP microstrip antenna for WLAN.

\section{MEASUREMENT}

The received power measurements conducted in the acechoic chamber were done by stepping up values of distance staring from $3 \mathrm{~cm}$ to $25 \mathrm{~cm}$. For the LTE microstrip antenna pairs, the operating frequency of $1.88 \mathrm{GHz}$ was used. For the WLAN microstrip antenna pairs, the operating frequency of $2.45 \mathrm{GHz}$ was used in the measurement. The proposed antennas are tested in an anechoic chamber. The measurement system is illustrated in Fig. 8. Both pairs of proposed antennas are used for transmitting and receiving power at $1.88 \mathrm{GHz}$ and $2.45 \mathrm{GHz}$ respectively. First, the first pair of antennas is placed in a fixed position, and its received power $P_{r}$ is measured through a spectrum analyzer by varying the distance from $3 \mathrm{~cm}$ to $25 \mathrm{~cm}$. Then, the second pair of antennas is replaced by the same procedure. By measuring the voltage across the resistor, the conversion efficiency can be obtained by equation (14),

$$
\eta(\%)=\frac{V_{L}^{2}}{R_{L}} \times \frac{1}{P_{\text {in }}} \times 100
$$

where $V_{L}$ is the voltage on the resistor, $R_{L}$ is the resistance value, and $P_{\text {in }}$ is the input power level sensed by the antenna.

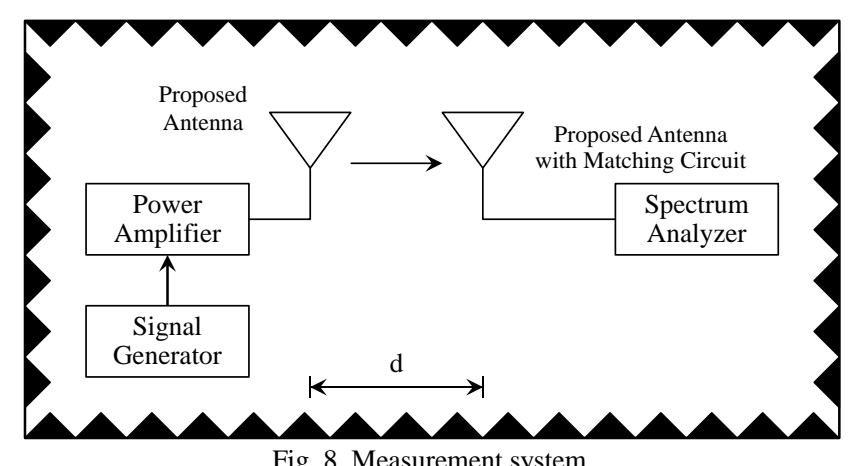

Transmitted power of $10 \mathrm{~mW}$ was adjusted for transmission and received power was measured by the power meter. Table IV and Fig. 9 show the results of the measurement. The conversion efficiency of the fabricated antennas was measured as $38 \%$ and $35 \%$.

\begin{tabular}{c|c|c} 
TABLE IV: RECEIVED POWER FROM MEASUREMENT \\
\hline \hline \multirow{3}{*}{ Distance $(\mathrm{cm})$} & \multicolumn{2}{|c}{ Power $(\mathrm{mW})$} \\
\cline { 2 - 3 } & LTE & WLAN \\
& $1.88 \mathrm{GHz}$ & $2.45 \mathrm{GHz}$ \\
\hline 3 & 3.800 & 3.500 \\
4 & 3.350 & 2.800 \\
5 & 3.000 & 2.650 \\
6 & 2.855 & 2.470 \\
7 & 2.500 & 2.015 \\
8 & 2.000 & 1.850 \\
9 & 1.785 & 1.500 \\
10 & 1.500 & 1.400 \\
11 & 1.320 & 1.300 \\
12 & 1.050 & 1.155 \\
13 & 0.895 & 0.975 \\
14 & 0.750 & 0.625 \\
15 & 0.600 & 0.575 \\
16 & 0.550 & 0.500 \\
17 & 0.490 & 0.450 \\
18 & 0.350 & 0.335 \\
19 & 0.250 & 0.245 \\
20 & 0.100 & 0.100 \\
21 & 0.050 & 0.050 \\
22 & 0.005 & 0.005 \\
23 & 0.005 & 0.005 \\
24 & 0.005 & 0.005 \\
25 & 0.005 & 0.005 \\
\hline \hline
\end{tabular}




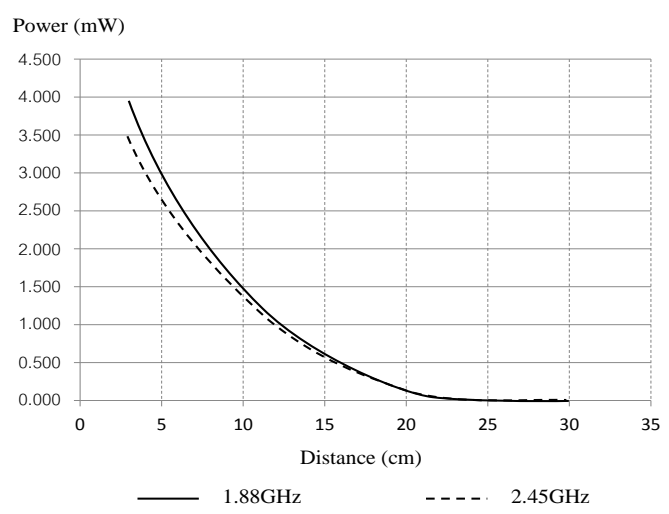

Fig. 9. Power received level of the proposed antennas.

\section{FUTURE WORK}

The rectenna circuit consists of a matching circuit and rectifying circuit as shown in Fig. 10. The rectifying circuit is a key element to improve the RF-DC conversion efficiency.

Therefore, for the future work, the different rectenna circuits will be designed and simulated by using ADS software. The software is used to evaluate and compare the performance of the various designs.

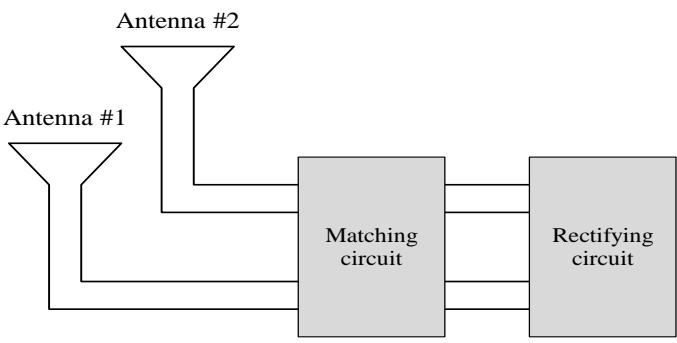

Fig. 10. The diagram of rectenna circuit.

\section{CONCLUSION}

Wireless Power Transfer System (WPT) has the potential to revolutionize the use of mobile devices by eliminating the need for a physical power supply and result in lowering cost for consumers. In this research, we proposed microstrip antenna design of rectenna for WPT system for LTE/WLAN with resonant frequency of $1.88 \mathrm{GHz}$ (TDD-LTE band number 35) and another rectenna with resonant frequency of $2.45 \mathrm{GHz}$ (ISM band). The proposed antenna with resonant frequency of $1.88 \mathrm{GHz}$ has $-24 \mathrm{~dB}$ return loss and antenna that resonates at $2.45 \mathrm{GHz}$ has $-25 \mathrm{~dB}$ return loss. The conversion efficiency of the fabricated antennas was measured as $38 \%$ and $35 \%$. In this research, we point out that the rectifying circuit is a key element to improve the RF-DC conversion efficiency. Additionally, for further research we propose that rectenna circuits be designed and simulated by using the ADS software which can evaluate and compare performance of the rectenna with different designs to conclude on the best design.

\section{REFERENCES}

[1] W. C. Brown, "The history of power transmission by radio waves," IEEE Trans. on Microwave Theory and Tech., pp. 1230-1242, 1984.

[2] G. Monti et al., "X-band planar rectenna," Antennas and Wireless Propagation Letters, vol. 10, pp. 1116-1119, 2011.

[3] F. E. Little, J. O. McSpadden, K. Chang, and N. Kaya, "Toward space solar power: Wireless energy transmission experiments past, present and future," in Proc. Space Technology and Applications International Forum, 1998, pp. 1225-1233.

[4] S. Pradhan, L. S.-K. Noh, and D.-Y. Choi, "Comparative study of rectenna for electromagnetic energy harvesting," International Journal of Control and Automation, vol. 7, pp. 101-112.

[5] T. Paing et al., "Wirelessly-powered wireless sensor platform," in Proc. European Conference on Wireless Technologies, 2007, pp. 241-244.

[6] Y. S. Khraisat, "Design of 4 elements rectangular microstrip patch antenna with high gain for $2.4 \mathrm{GHz}$ applications," Modern Applied Science, vol. 6, p. 68, 2012.

[7] R. Chen et al., "Design and experiment of a loop rectenna for RFID wireless power transmission and data communication applications," Progress in Electromagnetics Research Proceeding, 2009.

[8] M. E. Ahmad, "Energy harvesting using a cheap easy-to-fabricate FM rectenna," in Proc. 2009 World Congress on Electronics and Electrical Engineering WCEEENG, 2009, pp. 6-9.

[9] G. A. Vera, "Efficient rectenna design for ambient microwave energy recycling," 2009.

[10] J. Heikkinen et al., "Low-profile circularly polarized rectifying antenna for wireless power transmission at $5.8 \mathrm{GHz}$," Microwave and Wireless Components Letters, vol. 14, pp. 162-164, 2004.

[11] Y.-Y. Gao et al., "A circularly polarized rectenna with low profile for wireless power transmission," Progress In Electromagnetics Research Letters, vol. 13, pp. 41-49, 2010.

[12] D.-G. Fang, Antenna Theory and Microstrip Antennas, CRC Press, 2009.

[13] S. Malisuwan, J. Sivaraks, and N. Suriyakrai, "The performance of rectangular microstrip structure by applying the cole-cole diagram concept," Lecture Notes on Software Engineering, vol. 2, p. 48, 2014.

[14] R. Garg, Microstrip Antenna Design Handbook, Artech House, 2001.

[15] I. Poole, "LTE frequency bands \& spectrum allocations : A summary and tables of the LTE frequency band spectrum allocations for $3 \mathrm{G} \&$ 4G LTE - TDD and FDD," 2015.

[16] C. A. Balanis, Antenna Theory: Analysis and Design, John Wiley \& Sons, 2012.

[17] R. A. Sainati, CAD of Microstrip Antennas for Wireless Applications, Artech House, Inc., 1996.

[18] K. Odeyemi, D. Akande, and E. Ogunti, "Design of an S-band rectangular microstrip patch antenna," European Journal of Scientific Research, vol. 55, pp. 72-79, 2011.

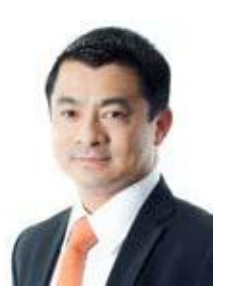

Settapong Malisuwan was born on March 24, 1966 in Bangkok, Thailand. He received his $\mathrm{PhD}$ degree in electrical engineering (telecommunications), specializing in mobile communication systems from Florida Atlantic University, Boca Raton in 2000. He received his MSc degree in electrical engineering in mobile communications system, from George Washington University in 1996, and MSc degree in electrical engineering in telecommunication engineering from Georgia Institute of Technology in 1992. He served in the Royal Thai Armed Forces for more than 25 years and is currently the vice chairman of National Broadcasting and Telecommunications and the chairman of Telecommunication Commission Bangkok, Thailand. His research interests are in electromagnetics, efficient spectrum management and telecommunications policy and management.

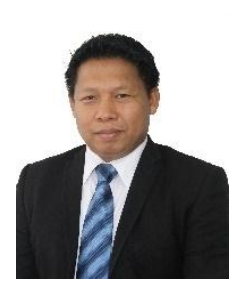

Noppadol Tiamnara was born on November 12, 1968 in Pah Na Korn Sri Ayuttaya, Thailand. He received his $\mathrm{BSc}$ degree in electrical engineering from Saint John's University, Thailand, 2002. He received his MSc degree in technology management from Thammasart University, Thailand, 2012. Since 2006, he has been working in National Broadcasting and Telecommunications Commission as the assistant to secretary of vice chairman of National Broadcasting and Telecommunication Commission (NBTC). His research interests include LTE design, wireless systems, microstrip antenna and applied electromagnetic.

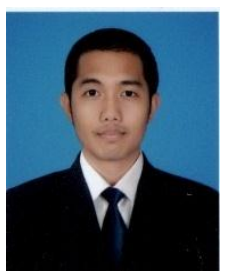

Nattakit Suriyakrai was born in Khonkhaen, Thailand on March 22, 1987. He received his bachelor of liberal arts in Japanese language from Thammasat University in 2010. He has been working as an assistant to vice chairman in National Broadcasting and Telecommunications, Bangkok, Thailand since November 2012. His research interests are in technology management and spectrum management. 